\title{
The Middle Mile: The Role of the Public Library in Ensuring Access to Broadband
}

This paper discusses the role of the public library in ensuring access to the broadband communication that is so critical in today's knowledge-based society. It examines the culture of information in 2010, and then asks what it means if individuals are online or not. The paper also explores current issues surrounding telecommunications and policy, and finally seeks to understand the role of the library in this highly technological, perpetually connected world.

西 $\mathrm{n}$ the last twenty years library collections have evolved from being predominantly print-based to ones that have a significant digital component. This trend, which has a direct impact on library services, has only accelerated with the advent of Web 2.0 technologies and participatory content creation. Cutting-edge libraries with next generation catalogs encourage patrons to post reviews, contribute videos, and write on library blogs and wikis. Even less adventuresome institutions offer a variety of electronic databases licensed from multiple publishers and vendors. The piece of these library portfolios that is at best ignored and at worst vilified is the infrastructure that enables Internet connectivity. In 2010, broadband telecommunication is recognized as essential to access the full range of information resources. Telecommunications experts articulate their concerns about the digital divide by focusing on first- and last-mile issues of bringing fiber and cable to end users. The library, particularly the public library, represents the metaphorical middle mile providing the public with access to rich information content. Equally important, it provides technical knowledge, subject matter expertise, and general training and support to library users.

This paper discusses the role of the public library in ensuring access to the broadband communication that is so critical in today's knowledge-based society. It examines the culture of information in 2010, and then asks what it means if individuals are online or not. The paper also explores current issues surrounding telecommunications and policy, and finally seeks to understand the role of the library in this highly technological, perpetually connected world.

\section{The Culture of Information}

Information today is dynamic. As the Internet continues on its fast paced, evolutionary track, what we call 'information' fluctuates with each emerging Web-based technology. Theoretically a democratic platform, the Internet and its user-generated content is in the process of fundamentally altering culture and society. In some circles the changes happen in real time as new Web-based applications are developed, adopted, and integrated into the user's daily life. These users are the early adopters; the Internet cognoscenti. Second tier users appreciate the availability of online resources and use a mix of devices to access Internet content but vary in the extent to which they try the latest application or device. The third tier users also vary in the amount they access the Internet but have generally not embraced its full potential, from not seeking out readily available resources to not connecting at all. ${ }^{1}$ Regardless of the degree to which they access the Internet, all of these users require basic technology skills and a robust underlying infrastructure.

Since the introduction of Web 2.0, the number and type of participatory Web-based applications has continued to grow. Many people are eagerly taking part in creating an increasing variety of Web-based content because the basic tools to do so are widely available. The amateur, creating and sharing for primarily personal reasons, has the ability to reach an audience of unprecedented size. In turn, the Internet audience, or virtual audience, can select from a vast menu of formats, including multimedia and print. With print resources disappearing, it is increasingly likely for an individual to only be able to access necessary material online. Web-based resources are unique in that they enable an undetermined number of people, personally connected or complete strangers, to interact with and manipulate the content thereby creating something new with each interaction and subsequent iteration. Many of these new resources and applications require much more bandwidth than traditional print resources. With the necessary technology no longer out of reach, a crosssection of society is affecting the course the twenty-first century is taking vis à vis how information is created, who can create it, and how we share it. ${ }^{2}$ In turn, who can access Web-based content and who decides how it can be accessed become critical questions to answer. As people become more adept at using Web-based tools and eager to try new applications, the need for greater broadband will intensify.

The economic downturn is having a marked effect on people's Internet use. If there was a preexisting problem with inadequate access to broadband, current circumstances exacerbate it to where it needs immediate attention. Access to broadband Internet today increases

Marijke Visser (mvisser@alawash.org) is Information Technology Policy Analyst and Mary Alice Ball (maryaliceball@yahoo .com) former Chair, Telecommunications Subcommittee, Office for Information Technology Policy, American Library Association, Washington, DC. 
the amount of information and variety of formats available to the user. In turn more content is being distributed as users create and share original content. ${ }^{3}$ Businesses, nonprofits, municipal agencies, and educational institutions appreciate that by putting their resources online they reach a broader segment of their constituency. This approach to reaching an audience works provided the constituents have their own access to the materials, both physically and intellectually. It is one thing to have an Internet connection and another to have the skill set necessary to make productive use of it.

As reported in Job-Seeking in U.S. Public Libraries in 2009 , "less than $44 \%$ of the top 100 U.S. retailers accept instore paper applications." ${ }^{4}$ Municipal, state, and federal agencies are increasingly putting their resources online, including unemployment benefit applications, tax forms, and court documents. ${ }^{5}$ In addition to online documents, the report finds social service agencies may encourage clients to make appointments and apply for state jobs online. ${ }^{6}$ Many of the processes that are now online require an ability to navigate the complexities of the Internet at the same time as navigating difficult forms and websites. The combination of the two can deter someone from retrieving necessary resources or successfully completing a critical procedure.

While early adopters and policy-makers debate the issues surrounding Internet access, the other strata of society, knowingly or not and to varying degrees, are enmeshed in the outcomes of these ongoing discussions because their right to information is at stake.

\section{Barriers to Broadband Access}

By condensing Internet access issues to focus on the availability of adequate and sustainable broadband, it is possible to pinpoint four significant barriers to access: price, availability, perceived relevance, and technical skill level. The first two barriers are determined by existing telecommunications infrastructure as well as local, state, and federal telecommunications policies. The latter barriers are influenced by individual behaviors. Both divisions deserve attention.

If local infrastructure and the Internet service provider (ISP) options do not support broadband access to all areas within its boundaries, the result will be that some community members can have broadband services at home while others must rely on work or public access computers. It is important to determine what kind of broadband services are available (e.g., cable, DSL, fiber, satellite) and if they are robust enough to support the activities of the community. Infrastructure must already be in place or there must be economic incentive for ISPs to invest in improving current infrastructure or in installing new infrastructure.
The geographical location of a community will also influence what kind of Internet service is available because of deployment costs. These costs are typically reflected in varying prices to consumers. In addition to the physical layout of an area, current federal telecommunications policies limit the degree to which incentives can be used on the local level. ${ }^{7}$ Encouraging competition between ISPs, including municipal electric utilities, incumbent local exchange carriers, and national cable companies, for example, requires coordination between local needs and state and federal policies. Such coordinated efforts are inherently difficult when taking into consideration the numerous differences between locales. Ultimately, though, all of these factors influence the price end users must pay for Internet access.

With necessary infrastructure and telecommunications policies in place, there are individual behaviors that also affect broadband adoption. According to the Pew study, "Home Broadband Adoption 2008," 62 percent of dial-up users are not interested in switching to broadband. ${ }^{8}$ Clearly there is a segment of the population that has not yet found personal relevance to high-speed access to online resources. In part this may be because they only have experience with dial-up connections. Depending on dial-up gives the user an inherently inferior experience because bandwidth requirements to download a document or view a website with multimedia features automatically prevent these users from accessing the same resources as a user with a high-speed connection. A dial-up user would not necessarily be aware of this difference. If this is the only experience a user has it might be enough to deter broadband adoption, especially if there are other contributing factors like lack of technical comfort or availability of relevant content.

Motivation to use the Internet is influenced by the extent to which individuals find content personally relevant. Whether it is searching for a job and filling out an application, looking at pictures of grandchildren, using Skype to talk to a family member deployed in Iraq, researching healthcare providers, updating a personal webpage, or streaming video, people who do these things have discovered personally relevant Internet content and applications. Understanding the potential relevance of going online makes it more likely that someone would experiment with other applications, thus increasing both the familiarity with what is available and the comfort level with accessing it. Without relevant content, there is little motivation for someone not inclined to experiment with Internet technology to cross what amounts to a significant hurdle to adoption. Anthony Wilhelm argues in a 2003 article discussing the growing digital divide that culturally relevant content is critical in increasing the likelihood that non-users will want to access Web-based resources. ${ }^{9}$ The scope of the issue of providing culturally relevant content is underscored in the 2008 Pew study, 
which found that of the 27 percent of adult Americans who are not Internet users, 33 percent report they are not interested in going online. ${ }^{10}$ That Pew can report similar information five years after the Wilhelm article identifies a barrier to equitable access that has not been adequately resolved.

\section{Models for Sustainable Broadband Availability}

In discussing broadband, the question of what constitutes broadband inevitably arises. Gillett, Lehr, and Osoria, in "Local Government Broadband Initiatives," offers a functional definition: "access is 'broadband' if it represents a noticeable improvement over standard dial-up and, once in place, is no longer perceived as the limiting constraint on what can be done over the Internet."11 While this definition works in relationship to dial-up, it is flexible enough to apply to all situations by focusing on "a noticeable improvement" and "no longer perceived as the limiting constraint" (added emphasis). Ensuring sustainable broadband access necessitates anticipating future demand. Short sighted definitions, applicable at a set moment in time, limit long-term viability of alternative solutions.

Devising a sustainable solution calls for careful scrutiny of alternative models, because the stakes are so high in the broadband debate. There are many different players involved in constructing information policies. This does not mean, however, that their perspectives are mutually exclusive. In debates with multiple perspectives, it is important to involve stakeholders who are aligned with the ultimate goal: assuring access to quality broadband to anyone going online.

What is successful for one community may be entirely inappropriate in another; designing a successful system requires examining and comparing a range of scenarios. Existing circumstances may predetermine a particular starting point, but one first step is to evaluate best practices currently in place in a variety of communities to come up with a plan that meets the unique criteria of the community in question. Sustainable broadband solutions need to be developed with local constituents in mind and successful solutions will incorporate the realities of current and future local technologies and infrastructure as well as local, state, and federal information policies.

Presupposing that the goal is to provide the community with the best possible option(s) for quality broadband access, these are key considerations to take into account when devising the plan. In addition to the technological and infrastructure issues, within a community there will be a combination of ways people access the Internet. There will be those who have home access, those who need public access, and those who do not seek access at all. Success hinges on understanding that each community is unique, on leveraging its strengths, and on ameliorating its weaknesses.

Local government can play a significant role in the availability of broadband access. From a municipal perspective, emphasizing the role of broadband as a factor in economic development can help define how the municipality should most effectively advocate for broadband deployment and adoption. Gillett offers four initiatives appropriate for stimulating broadband from a local viewpoint. Municipal governments can

- become leaders in developing locally relevant Internet content and using broadband in their own services;

- adopt policies that make it easier for ISPs to offer broadband;

- subsidize broadband users and/or ISPs; or

- become involved in providing the infrastructure or services themselves. ${ }^{12}$

Individually or in combination these four initiatives underscore the fact that government awareness of the possibilities for community growth made possible by broadband access can lead to local government support for the initiatives of other local agencies, including nonprofit, municipal, or small businesses. Agencies partnering to support community needs can provide evidence to local policy makers that broadband is essential for community success. Once the municipality sees the potential for social and economic development, it is more likely to support policies that stimulate broadband buildout.

Building strong local partnerships will set the stage for the development of a sustainable broadband initiative as the different stakeholders share perspectives that take into account a variety of necessary components. When the time comes to implement a strategy, not only will different perspectives have been included, the plan will have champions to speak for it: the government, ISPs, public and private agencies, and community members. It is important to know which constituents are already engaged in supporting community broadband initiatives and which should be tapped. The ultimate purpose in establishing broadband Internet access in a community is to benefit the individual community members, thereby stimulating local economic development. Key players need to represent agencies that recognize the individual voice.

A 2004 study led by Strover provides an example of the importance of engaging local community leaders and agencies in developing a successful broadband access project. ${ }^{13}$ The study looked at thirty-six communities that received state funding to establish community technology centers (CTC). It addressed the effective use and management of CTCs and called attention to the inadequacy of supplying the hardware without community support 
systems in place. Users need a support system that highlights opportunities available via the Internet and that provides help when they run into problems. Access is more than providing the infrastructure and hardware. The potential users must also find content that is culturally relevant in an environment that supports local needs and expectations. Strover found the most successful CTCs were located in places that "actively attracted people for other social and entertaining reasons." ${ }^{14}$ In other words, the CTCs did not operate in a vacuum devoid of social context. Successful adoption of the CTCs as a resource for information was dependent on the targeted population finding culturally relevant content in a supportive environment. An additional point made in the study showed that without strong community leadership, there was not significant use of the CTC even when placed in an already established community center. ${ }^{15}$ This has significant implications for what constitutes access as libraries plan broadband initiatives.

Investments in technology and a national commitment to ensure universal access to these new technologies in the 1990s provide the current policy framework. As suggested by Wilhelm in 2003, to continue to move forward the national agenda needs to focus on updating policies to fit new information circumstances as they arise. Today's information policy debates should emphasize a similar focus. Beyond accelerating broadband deployment into underserved areas, Wilhelm suggests there needs to be support for training and content development that guarantees communities will actually use and benefit from having broadband deployed in their area. ${ }^{16}$ Technology training and support for local agencies that provide the public with Internet access, as well as opportunities for the individuals themselves, is essential if policies are going to actually lead to useful broadband adoption. Individual and agency Internet access and adoption require investment beyond infrastructure; they depend on having both culturally relevant content and the information literacy skills necessary to benefit from it.

\section{Finding the Right Solution}

Though it may have taken an economic crisis to bring broadband discussions into the living room, the result is causing renewed interest in a long-standing issue. Many states have formed broadband task forces or councils to address the lack of adequate broadband access at the state level and, on the national front, broadband was a key component of the American Recovery and Reinvestment Act of 2009. ${ }^{17}$ The issue changes as technologies evolve but the underlying tenet of providing people access to the information and resources they need to be productive members of society is the same. What becomes of the current emphasis on universal broadband depends on selecting the best of the alternative plans according to carefully vetted criteria in order to develop a flexible and forward-thinking course of action.

Can we let people remain without access to robust broadband and the necessary skill set to use it effectively? No. As more and more resources critical to basic life tasks are accessible only online, those individuals that face challenges to going online will likely be socially and economically disadvantaged when compared to their online counterparts. Recognition of this potential for intensifying digital divide is recognized in the Federal Communication Commission's (FCC) National Broadband Plan (NBP) released in March 2010. ${ }^{18}$ The NBP states six national broadband goals, the third of which is "Every American should have affordable access to robust broadband service, and the means and skills to subscribe if they so choose." ${ }^{19}$ Research conducted for the recommendations in the NBP was comprehensive in scope including voices from industry, public interest, academia, and municipal and state government. Responses to more than thirty public notices issued by the FCC provide evidence of wide concern from a variety of perspectives that broadband access should become ubiquitous if the United States is to be a competitive force in the twentyfirst century.

Access to essential information such as government, public safety, educational, and economic resources requires a broadband connection to the Internet. It is incumbent on government officials, ISPs, and community organizations to share ideas and resources to achieve a solution for providing their communities with robust and sustainable broadband. It is not necessary to have all users up to par with the early adopters. There is not a one-size-fits-all approach to wanting to be connected, nor is there a one-size-fits-all solution to providing access. What is important is that an individual can go online via a robust, high-speed connection that meets that individual's needs at that moment.

What this means for finding solutions is

- there needs to be a range of solutions to meet the needs of individual communities;

- they need to be flexible enough to meet the evolving needs of these communities as applications and online content continue to change; and

- they must be sustainable for the long term so that the community is prepared to meet future needs that are as yet unknown.

Solutions to providing broadband Internet access will be most successful when they are designed starting at the local level. Community needs vary according to local demographics, geography, existing infrastructure, types of service providers, and how state and federal 
policies mesh with local ordinances. Local stakeholders best understand the complex interworking of their community and are aware of who should be included in the decision-making process. Including a local perspective will also increase the likelihood that as community needs change, new issues will be brought to the attention of policy makers and agencies who advocate for the individual community members.

Community agencies that already are familiar with local needs, abilities, and expectations are logical groups to be part of developing a successful local broadband access strategy. The library exemplifies a community resource whose expertise in local issues can inform information policy discussions on local, state, and federal levels. As a natural extension of library service, libraries offer the added value support necessary for many users to successfully navigate the Internet. The library is an established community hub for informational resources and provides dedicated staff, technology training opportunities, and no-fee public access computers with an Internet connection. Libraries in many communities are creating locally relevant Web-based content as well as linking to other community resources on their own websites. Seeking a partnership with the local library will augment a community broadband initiative.

It is difficult to appreciate the impacts of current information technologies because they change so rapidly there is not enough time to realistically measure the effects of one before it is mixed in with a new innovation. With Web-based technologies there is a lag time between what those in the front of the pack are doing online and what those in the rear are experiencing. While there is general consensus that broadband Internet access is critical in promoting social and economic development in the twenty-first century as is evidenced by the national purposes outlined in the NBP, there is not necessarily agreement on benchmarks for measuring the impacts. Three anticipated outcomes of providing community access to broadband are

- civic participation will increase;

- communities will realize economic growth; and

- individual quality of life will improve.

When a strategy involves significant financial and energy investments there is a tendency to want palpable results. The success of providing broadband access in a community is challenging to capture. To achieve a level of acceptable success it is necessary to focus on local communities and aggregate anecdotal evidence of incremental changes in public welfare and economic gain. Acceptable success is subjective at best but can be usefully defined in context of local constituencies.

Referring to participation in the development of a vibrant culture, Horrigan notes that "while inherently difficult to measure, these kinds of social and cultural capital are important elements in ongoing debates about uses and consequences of broadband access. An ongoing challenge for those interested in the social, economic, and policy consequences of modern information networks will be to keep up with changing notions of what it means to be connected in cyberspace." 20

The social contexts in which a broadband plan will be enacted influence the appropriateness of different scenarios and should help guide which ones are implemented. Engaging a variety of stakeholders will increase the likelihood of positive outcomes as community members embrace the opportunities provided by broadband Internet access. It is difficult, however, to anticipate the outcomes that may occur as users become more familiar with the resources and achieve a higher level of comfort with technology. Ramirez states,

The "unexpected outcomes" section of many evaluation reports tends to be rich with anecdotes.... The unexpected, the emergent, the socially constructed innovations seem to be, to a large extent, off the radar screen, and yet they often contain relevant evidence of how people embrace technology and how they innovate once they discover its potential. ${ }^{21}$

Community members have the most to gain from having broadband Internet access. Including them will increase the community's return on its investment as they take advantage of the available resources. Ramirez suggests that "participatory, learning, and adaptive policy approaches" will guide the community toward developing communication technology policies that lead to a vibrant future for individuals and community alike. ${ }^{22}$ As success stories increase, the aggregation of local communities' social and economic growth will lead to a net sum gain for the nation as a whole.

\section{The Role of the Library}

Public libraries play an important role in providing Internet access to their community members. According to a 2008 study, the public library is the only outlet for no-fee Internet access in 72.5 percent of communities nationwide; in rural communities the number goes up to 82.0 percent. ${ }^{23}$ Beyond having desktop or, in some cases, wireless access, public libraries offer invaluable user support in the form of technical training and locally relevant content. Libraries provide a secondary community resource for other local agencies who can point their clients to the library for no-fee Internet access. In today's economy where anecdotal reports show an increase in library use, particularly Internet use, the role of the public 
library as a stable Internet provider cannot be overestimated. To maintain its vital function, however, the library must also resolve infrastructure challenges of its own. Because of the increased demand for access to Internet resources, public libraries are finding their current broadband services are not able to support the demand of their patrons. The issues are two-fold: increased patron use means there are often neither sufficient workstations nor broadband speeds to meet patron demand. In 2008, about 82.5 percent of libraries reported an insufficient number of public workstations, and about 57.5 percent reported insufficient broadband speeds. ${ }^{24}$ To add to these already significant issues, the report indicates libraries are having trouble supporting the necessary information technology (IT) because of either staff time constraints or the lack of a dedicated IT staff. ${ }^{25}$ Public libraries are facing considerable infrastructure management issues at a time when library use is increasing. Overcoming the challenges successfully will require support on the local, state, and federal level.

Here is where the librarian, as someone trained to become inherently familiar with the needs of her local constituency and ethically bound to provide access to a variety of information resources, needs to insert herself into the debate. Librarians need to be ahead of the crowd as the voice that assures content will be readily accessible to those who seek it. Today, the elemental policy issue regarding access to information via the Internet hinges on connectivity to a sustainable broadband network. To promote equitable broadband access, the librarian needs be aware of the pertinent information policies in place or under consideration, and be able to anticipate those in the future. Additionally, she will need to educate local policy makers about the need for broadband in their community. In some circumstances, the librarian will need to move beyond her local community and raise awareness of community access issues on the state and federal level. The librarian is already able to articulate numerous issues to a variety of stakeholders and can transfer this skill to advocate for sustainable broadband strategies that will succeed in her local community.

There are many strata of Internet users, from those in the forefront of early adoption to those not interested in being online at all. The early adopters drive the market which responds by making resources more and more likely to be primarily available only online. As we continue this trend, the social repercussions increase from merely not being able to access entertainment and news to being unable to participate in the knowledge-based society of the twenty-first century. By folding in added value online access for the community, the library helps increase the likelihood that the community will benefit from broadband being available to the library patrons and by extension to the community as a whole. To realize the Internet's full potential, access to it cannot be provided in isolation. An individual must possess skills to navigate the online resources. As users gain an understanding of the potential personal growth and opportunities broadband yields, they will be more likely to seek additional online resources. By stimulating broadband use, the library will contribute to the social and economic health of the community.

If the library is to extend its role as the information hub in the community by providing no-fee access to broadband to anyone who walks through the door, the local community must be prepared to support that role. It requires a commitment to encourage build out of appropriate technology necessary for the library to maintain a sustainable Internet connection. It necessitates that local communities advocate for national information and communication policies that are pro-library. When public policy supports the library's efforts, the local community benefits and society at large can progress.

What if the library's own technology needs are not met? The role of the library in its community is becoming increasingly important as more people turn to it for their Internet access. Without sufficient revenue, the library will have a difficult time meeting this additional demand for services. In turn, in many libraries increased demand for broadband access stretches the limit of IT support for both the library staff and the patrons needing help at the computers. What will be the fallout from the library not being able to provide Internet services the patrons desire and require? Will there be a growing skills difference between people who adopt emerging technologies and incorporate them into their daily lives and those who maintain the technological status quo? What will the social impact be of remaining off line either completely or only marginally? Can the library be the bridge between those on the edge, those in the middle, and those at the end? With a strong and well articulated vision for the future, the library can be the link that provides the community with sustainable broadband.

\section{Conclusion}

The recent national focus on universal broadband access has provided an opportunity to rectify a lapse in effective information policy. Whether the goal includes facilitating meaningful access continues to be more elusive. As government, organizations, businesses, and individuals rely more heavily on the Internet for sharing and receiving information, broadband Internet access will continue to increase in importance. Following the status quo will not necessarily lead to more people having broadband access in the long run. The early adopters will continue to stimulate technological innovation which, in turn, will trickle down the ranks of the different user types. Currently, 
however, the supply of Internet resources is unevenly stimulating user demand and the unequal distribution of broadband access has greater potential for significant negative social consequences. Staying the course and following a haphazard evolution of broadband adoption, may, in fact, renew valid concerns about a digital divide. Without an intentional and coordinated approach to developing a broadband strategy, its success is likely to fall short of expectations. The question of how to ensure that Internet content is meaningful requires instituting a plan on a very local level, including stakeholders who are familiar with the unique strengths and weaknesses of their community.

Strover, in her 2000 article The First Mile, suggests connectivity issues should be viewed from a first mile perspective where the focus is on the person accessing the Internet and her qualitative experience rather than from a last mile perspective which emphasizes ISP, infrastructure, and market concerns. ${ }^{26}$ Both perspectives are talking about the same physical section of the connection network: the piece that connects the user to the network. According to Strover, distinguishing between the first mile and last mile perspectives is more than an arbitrary argument over semantics. Instead, a first mile perspective represents a shift "in the values and priorities that shape telecommunications policy. ${ }^{\prime 27}$ By switching to a first mile perspective, connectivity issues immediately take into account the social aspects of what it means to be online. Who will bring this perspective to the table? And how will we ascertain what the best approach to supporting the individual voice should be?

The first mile perspective is one the library is intimately familiar with as an organization that traditionally advocates for the first mile of all information policies. The library is in a key position in the connectivity debate because of its inclination to speak for the user and to be aware of the unique attributes and needs of its local community. As part of its mission, the library takes into account the distinctive needs of its user community when it designs and implements its services. A natural outgrowth of this practice is to be keenly aware of the demographics of the community at large. The library can leverage its knowledge and understanding to create an even greater positive impact on the social, educational, and economic community development made possible by broadband adoption. To extend the first mile perspective analogy, in the connectivity debate, the library will play the role of the middle mile: the support system that successfully connects the Internet to the consumer.

While the target populations for stimulating demand for broadband are really those in the second tier of users, by advocating for the first mile perspective, the library will be advocating for equitable information policies whose implementation has bearing on the early adopters as well. By stimulating demand for broadband within a community, the entire community benefits regardless of where and how the individuals go online.

The effects of the Internet are now becoming broadly social enough that there is a general awareness that the Internet is not decoration on contemporary society but a challenge to it. ${ }^{28}$

Being connected is no longer an optional luxury; to engage in the twenty-first century it is essential. Access to the Internet, however, is more than simple connectivity. Successful access requires: an understanding of the benefits to going on line, technological comfort, information literacy, ongoing support and training, and the availability of culturally relevant content. People are at various levels of Internet use, from those eagerly anticipating the next iteration of Web-based applications to those hesitant to open an e-mail account. This user spectrum is likely to continue. Though the starting point may vary depending on the applications that become important to the user in the middle of the spectrum, there will be those out in front and those barely keeping up.

The implications of the pervasiveness of the Internet are only beginning to be appreciated and understood. Because of their involvement at the cutting edge of Internet evolution, librarians can help lead the conversations. Libraries have always been situated in neutral territory within their communities and closely aligned with the public good. Librarians understand the perspective of their patrons and are grounded in their local communities. Librarians can therefore advocate effectively for their communities on issues that may not completely be understood or even recognized as mattering. Connectivity is an issue supremely important to the library as today access to the full range of information necessitates a broadband connection.

Libraries have carved out a role for themselves as a premier Internet access provider in the continually evolving online culture. As noted by Bertot, McClure, and Jaeger, the "role of Internet access provider for the community is ingrained in the social perceptions of public libraries, and public Internet access has become a central part of community perceptions about libraries and the value of the library profession." 29

In times of both economic crisis and technological innovation, there are many unknowns. In part because of these two juxtaposed events, the role of the public library is in flux. Additionally, the network of community organizations that libraries link to is becoming more and more complex. It is a time of great opportunity if the library can articulate its role and frame it in relationship to broader society. Evolving Internet applications require increasing amounts of bandwidth and the trend is to make these bandwidth-heavy applications more and more vital to daily life. One clear path the library community can take 
is to develop its role as the middle mile connecting the increasing breadth of Internet resources to the general public. The broadband debate has moved out of the background of telecommunication policy and into the center of public attention. Now is the moment that calls for an information policy advocate who can represent the end user while understanding the complexity of the other stakeholder perspectives. The library undoubtedly has its own share of stakeholders, but over time it is an institution that has maintained a neutral stance within its community, thereby achieving a unique ability to speak for all parties. Those who speak for the library are able to represent the needs of the public, work with a diverse group of stakeholders, and help negotiate a sustainable strategy for providing broadband Internet access.

\section{References and notes}

1. Lee Rainie, " 2.0 and the Internet World," Internet Librarian 2007, http://www.pewinternet.org/Presentations/2007/20 -and-the-Internet-World.aspx (accessed Mar. 4, 2009). See also John Horrigan, "A Typology of Information and Communication Technology Users," 2007, www.pewinternet.org/ /media// Files/Reports/2007/PIP_ICT_Typology.pdf.pdf (accessed Feb. 12, 2009).

2. Lawrence Lessig, "Early Creative Commons History, My Version," video blog post, 2008, http://lessig.org/ blog/2008/08/early_creative_commons_history.html (accessed Jan. 20, 2009). See the relevant passage from 20:53 through 21:50.

3. John Horrigan, "Broadband: What's All the Fuss About?" 2007, p. 1, http://www.pewinternet.org/ /media/ Files/Reports/2007/BroadBand\%20Fuss.pdf.pdf (accessed Feb. 12, 2009).

4. "Job-Seeking in US Public Libraries," Public Library Funding \& Technology Access Study, 2009, http://www.ala.org/ ala/research/initiatives/plftas/issuesbriefs/brief_jobs_july.pdf (accessed Mar. 27, 2009).

5. Ibid.

6. Ibid.

7. Sharon E. Gillett, William H. Lehr, and Carlos Osorio, "Local Government Broadband Initiatives," Telecommunications Policy 28 (2004): 539.

8. John Horrigan, "Home Broadband Adoption 2008," 10, http:/ / www.pewinternet.org/ / media / /Files/Reports /2008/ PIP_Broadband_2008.pdf (accessed Feb. 12, 2009).

9. Anthony G. Wilhelm, "Leveraging Sunken Investments in Communications Infrastructure: A Policy Perspective from the United States," The Information Society 19 (2003): 279-86.

10. Horrigan, "Home Broadband Adoption," 12.

11. Gillett, Lehr, and Osorio, "Local Government Broadband
Initiatives," 538.

12. Ibid., 537-58.

13. Sharon Strover, Gary Chapman, and Jody Waters, "Beyond Community Networking and CTCs: Access, Development, and Public Policy," Telecommunications Policy 28, no. 7/8 (2004): 465-85.

14. Ibid., 483.

15. Ibid.

16. Wilhelm, "Leveraging Sunken Investments in Communications Infrastructure," 282.

17. See, for example, the Virginia Broadband Round Table (http:/ / www.otpba.vi.virginia.gov/broadband_roundtable .shtml), the Ohio Broadband Council (http://www.ohiobroad bandcouncil.org/), and the California Broadband Task Force (http://gov.ca.gov/speech/4596. See www.fcc.gov/recovery/ broadband/) for information on broadband initiatives in the American Recovery and Reinvestment Act.

18. Federal Communication Commission, National Broadband Plan: Connecting America, http:/ /www.broadband.gov/ (accessed Apr. 11, 2010).

19. Ibid.

20. Horrigan, "Broadband: What's All the Fuss About?" 2.

21. Ricardo Ramirez, "Appreciating the Contribution of Broadband ICT with Rural and Remote Communities: Stepping Stones toward and Alternative Paradigm," The Information Society 23 (2007): 86.

22. Ibid., 92.

23. Denise M. Davis, John Carlo Bertot, and Charles, R. McClure, "Libraries Connect Communities: Public Library Funding \& Technology Access Study 2007-2008," 35, http:// www.ala.org/ala/aboutala/offices/ors/plftas/0708/Libraries ConnectCommunities.pdf (accessed Jan. 24, 2009).

24. John Carlo Bertot et al., "Public Libraries and the Internet 2008: Study Results and Findings," 11, http:/ /www.ii.fsu.edu/ projectFiles/plinternet/2008/Everything.pdf (accessed Jan. 24, 2009). These numbers represent an increase from the previous year's study which suggests that libraries while trying to meet demand are not able to keep up.

25. Ibid.

26. Sharon Strover, "The First Mile," The Information Society 16 , no. 2 (2000): 151-54.

27. Ibid., 151.

28. Clay Shirky, "Here Comes Everybody: The Power of Organizing without Organizations." Berkman Center for Internet \& Society (2008). Video presentation. Available at http:// cyber.law.harvard.edu/interactive/events / 2008/02/shirky (Retrieved March 1, 2009).

29. John Carlo Bertot, Charles R. McClure, and Paul T. Jaeger, "The Impacts of Free Public Internet Access on Public Library Patrons and Communities," Library Quarterly 78, no. 3 (2008): 286, http:/ / www.journals.uchicago.edu.proxy.ulib.iupui.edu/ doi/pdf/10.1086/588445 (accessed Jan. 30, 2009). 